\title{
Variations in the mental health and sense of coherence (SOC) of new graduate nurses and the effects of SOC on variations in mental health
}

\author{
Tomoko Takeuchi ${ }^{1}$, Taisuke Togari ${ }^{2}$, Makoto Oe $^{3}$, Yukie Takemura ${ }^{4}$, Hiromi Sanada ${ }^{3}$ \\ ${ }^{1}$ Faculty of Nursing, Tokyo Healthcare University, Tokyo, Japan \\ ${ }^{2}$ Faculty of Liberal Arts, The Open University of Japan, Chiba, Japan \\ ${ }^{3}$ Graduate School of Medicine, The University of Tokyo, Tokyo, Japan \\ ${ }^{4}$ Research Hospital, The Institute of Medical Science, The University of Tokyo, Tokyo, Japan \\ Email: tomoko-t@umin.ac.jp
}

Received 30 January 2013; revised 6 March 2013; accepted 15 March 2013

\begin{abstract}
The current research conducted a longitudinal study of new graduate nurses at 3 times-prior to their employment as nurses and 3 months and 1 year afterward. This study yielded 3 findings. 1) The mental health of new graduate nurses significantly worsens 3 months after employment as a nurse and mental health 1 year after employment as a nurse was significantly worse than that before employment. 2) A sense of coherence (SOC) is the ability to cope with stressors, and the 3 components of SOC decreased significantly in the year after employment as a nurse. 3) The mental health of new graduate nurses was significantly affected by a low SOC and by previous diminishing of SOC. Findings suggested the importance of support to sustain and improve the mental health of new graduate nurses and the importance of approaches in the workplace to encourage the development of SOC.
\end{abstract}

Keywords: Longitudinal Study; Mental Health; New Graduate Nurses; Sense of Coherence (SOC)

\section{INTRODUCTION}

According to estimates from the Ministry of Health, Labour and Welfare [1], Japan is now faced with a shortage of nurses, and this problem is only expected to grow in the near future. New graduate nurses are a valuable human resource to compensate for the lack of nurses in clinical settings.

\subsection{New Graduate Nurses’ Mental Health}

The mental health of new graduate nurses in Japan is said to worsen particularly around June, i.e. 3 months after employment as a nurse [2-4]. Similarly, it is reported that depressive tendencies, obsessive compulsive disorder, and alcohol dependence increased from 1 to 3 months after employment as a nurse [5].

However, previous studies cited as evidence in Japan merely studied the worsening of new graduate nurses' mental health after their employment as nurses, so whether mental health had worsened upon employ mentor was poor to begin with was unclear. The mental health after employment as a nurse must be compared to its baseline level before employment as a nurse in order to obtain adequate evidence of the dynamic phenomenon of "worsening" of new graduate nurses' mental health.

\subsection{New Graduate Nurses' Ability to Cope with Stressors}

A number of previous studies have indicated that the level of occupational stressor is associated with new graduate nurses' poor mental health [6-8]. However, nursing is, to some extent, a profession in which occupational stressor cannot be avoided $[9,10]$, so there are limits on the extent to which occupational stressor can be reduced by external approaches alone. As indicated by Lazarus [11] in his cognitive appraisal model, what determines whether the relationship between a person and the environment is stressful depends on the individual's cognitive appraisal. Even if a nurse is exposed to occupational stressor, an external factor, to some extent, approaches affecting internal factors in new graduate nurses are important as they allow nurses to readily cope with that stressors and avoid a stress response [12].

Among the various concepts related to people's strategies to cope with stressors, a sense of coherence (SOC) is a particularly helpful one in terms of studying the health of workers. Such study requires a flexible approach affecting both internal and external factors, and the SOC is 
an extensively validated way to do so. SOC is a concept put forth by the sociologist Antonovsky and is defined as "a global orientation that expresses the extent to which one has a pervasive, enduring though dynamic feeling of confidence" [13]. This confidence relates to 3 types of perceptions of the stimuli deriving from one's internal and external environments in the course of living. SOC consists of 3 components. First is sense of comprehensibility, "the feeling of confidence that ... the stimuli deriving from one's internal and external environments in the course of living are structured, predictable and explicable". Second is sense of manageability, "the feeling of confidence that $\ldots$ the resources are available to one to meet the demands posed by these stimuli". Third is sense of meaningfulness, "the feeling of confidence that ... these demands are challenges, worthy of investment and engagement" [13]. People with a high SOC have confidence that allows them to find coherence in the world in which they live. Even in instances where that coherence is threatened by stressors, such people can readily cope with stressors and overcome stressful situations [13,14].

In the last few years, the importance of SOC has begun to attract attention in nursing management [15]. Nurses with a higher SOC have been found to have better mental health [16], less incidence of burnout [17,18], a higher level of job satisfaction [19], and a greater sense of achievement with regard to regular activities [20]. A high SOC has also been found to buffer the effects of work-family conflict on depression $[21,22]$. SOC, which indicates the ability to readily cope with stressors, is an important internal quality for new graduate nurses. However, there have been very few papers on the SOC of new graduate nurses. Thus, variations in the SOC of new graduate nurses before and after their employment as nurses must be studied and the effects of those variations on worsening mental health must be studied in order to determine the usefulness of fostering the internal qualities of new graduate nurses with regard to coping with stressors.

\subsection{Purpose}

The first aim of the current study was to describe actual variations in the mental health and SOC of new graduate nurses before and after their employment as nurses. The second aim was to determine the effects of variations in new graduate nurses' mental health on SOC.

The current study noted the importance of support to sustain and improve the mental health of new graduate nurses at medical facilities in Japan. This study also provides a basis to propose effective approaches to foster the internal qualities of new graduate nurses as a new path for that support.

\section{METHODOLOGY}

\subsection{Respondents}

Of nursing schools that were randomly selected, the facilities surveyed were 2 universities and 2 vocational schools in a major metropolitan area. Respondents were 258 students in their final year of study in January 2010 who planned to begin working as nurses in hospitals starting in April 2010. Respondents completed a selfadministered questionnaire 3 times (prior to employment as a nurse and 3 months and 1 year afterwards). Valid responses from 138 respondents who completed the questionnaire all 3 times were analyzed.

\subsection{Collection of Data}

\subsubsection{Survey before Employment as a Nurse (T1)}

In January 2010, the purpose and methodology of the study was explained to nursing students who qualified as participants. Consent was obtained from 256 students, and the students completed an anonymous self-administered questionnaire on paper. In order to facilitate subsequent follow-up, e-mail address was collected to allow respondents to be sent a questionnaire after their employment as nurses.

\subsubsection{Survey 3 Months after Employment as a Nurse} (T2)

A restricted online survey of the 204 nursing students who provided valid responses to the survey at $\mathrm{T} 1$ was conducted with an anonymous self-administered questionnaire in June 2010, 3 months after the new graduate nurses were employed in hospitals as nurses. Questionnaire links were sent to the e-mail address noted during the survey at $\mathrm{T} 1$.

\subsubsection{Survey 1 Year after Employment as a Nurse} (T3)

As was done at T2, a survey of the 204 individuals who provided valid responses to the survey at T1 was conducted with an anonymous self-administered questionnaire in March 2011 (T3), 1 year after their employment as nurses.

\subsection{Survey Items}

\subsubsection{Demographic Characteristics}

Respondents were asked their age, sex, marital status, whether or not they had children, and the level of their nursing education.

\subsubsection{Work Characteristics}

Respondents were asked about continued employment, the type of hospital where they worked, and the department to which they were assigned. Individuals who de- 
scribed their continued employment as something other than "continuing to work as a nurse" were asked when and why they no longer worked as a nurse.

\subsubsection{Mental Health}

The General Health Questionnaire (GHQ) was developed by Goldberg [23] to measure the mental health of the general population, and the reliability and validity of the 12-item GHQ have been verified in Japan [24,25]. Each question was answered on a 4-point scale, and responses were scored from 0 to 3 and tallied. A higher score indicates poorer mental health. Cronbach's $\alpha$ for scores at the 3 times in the current study ranged from 0.879 to 0.893 .

\subsubsection{SOC}

The SOC Scale was developed by Antonovsky [26], and the reliability and validity of the 13-item SOC scalehave been verified in Japan by Yamazaki [27]. (The examples of three sub scalesare as follows. Sense of comprehensibility; "Has it happened in the past that you were surprised by the behavior of people whom you knew well?", Sense of manageability; "Do you have any clear direction in your life?", Sense of meaningfulness; "Does that you carry out every day give you joy and satisfaction?") Each question was answered on a 7-point scale, and responses were scored from 1 to 7 and tallied. A higher score indicates a higher SOC. Cronbach's $\alpha$ for scores at the 3 times in the current study ranged from 0.749 to 0.764 .

\subsection{Analyses}

\subsubsection{Description of Variations in Mental Health and SOC upon Employment as a Nurse}

The mean GHQ and SOC scores at the 3 times from T1 to T3 were calculated, and analysis of variance (repeated measures) was performed using a general linear model with demographic characteristics as covariates. Afterwards, multiple comparison was done using Bonferroni's technique.

\subsubsection{Study of the Effects of SOC on Variations in Mental Health}

Multiple regression analysis was performed using variability in the GHQ score from $\mathrm{T} 1$ to $\mathrm{T} 2$ and from $\mathrm{T} 2$ to $\mathrm{T} 3$ as a dependent variable. Multi-collinearity was taken into account, and demographic characteristics and SOC scores at each time, and variability of SOC over time were entered as independent variables.

The above analysis was done using statistical software SPSS $20.0 \mathrm{~J}$ for Windows with a level of significance of $5 \%$ (two-sided).

\subsection{Ethical Considerations}

This study was approved by the Ethics Committee of the
Faculty and Graduate School of Medicine, The University of Tokyo (approval no. 2866) and research ethics committees of the facilities surveyed. Specifically, respondents were informed of the purpose of the study and that study participation was voluntary; respondents were also informed that whether or not they participated would have no effect whatsoever on their grades. In addition, careful attention was paid to data handling so that contact information would not be divulged.

\section{RESULTS}

\subsection{Number of Respondents and Their Responses}

At T1, 256 questionnaires were distributed and 208 responses were received (response rate: $81.3 \%$ ). Of these, 4 lacked vital data such as necessary contact information for follow-up or GHQ or SOC scores and were thus excluded, resulting in 204 valid responses (valid response rate: $79.7 \%$ ).

At T2 and T3, questionnaire links were sent to the 204 individuals who provided valid responses at $\mathrm{T} 1$. The online survey was configured to "check for required answers" to automatically detect if every question was answered and request that all required questions be answered, so the collected data consisted entirely of valid responses. The current study had 181 responses/valid responses $(88.7 \%)$ at $\mathrm{T} 2$ and $142(79.6 \%)$ at $\mathrm{T} 3$. There were 138 individuals who provided valid responses at all 3 times from $\mathrm{T} 1$ to $\mathrm{T} 3$, and these responses were analyzed in the current study.

\subsection{Respondent Characteristics (Table 1)}

Mean age was $24.0 \pm 2.7$ years at T1, and 138 respondents $(100 \%)$ were unmarried and had no children at all 3 times. The level of nursing education was university for 72 respondents (52.2\%) and vocational school for 66 $(47.8 \%)$. All of the respondents continued to work as nurses in the same department of the same facility at $\mathrm{T} 2$ and T3. The type of hospital where one worked was an advanced care hospital for 98 respondents (71.1\%), and all of these individuals were assigned to wards.

\subsection{Variations in Mental Health upon Employment as a Nurse (Table 2)}

The mean GHQ score was $16.4 \pm 6.2$ points at $\mathrm{T} 1,23.0 \pm$ 7.2 at $\mathrm{T} 2$, and $18.5 \pm 6.3$ at $\mathrm{T} 3$ (a higher GHQ score indicates poorer mental health). Multiple comparisons indicated that the mean at $\mathrm{T} 2$ was significantly higher $(\mathrm{p}<$ 0.001 ) than that at $\mathrm{T} 1$, and mental health significantly worsened 3 months after employment as a nurse. The mean GHQ score also decreased significantly $(\mathrm{p}<0.001)$ from $\mathrm{T} 2$ to $\mathrm{T} 3$, but the mean at $\mathrm{T} 3$ was significantly 
Table 1. Respondent charactaristics ${ }^{1} \mathrm{n}=138$.

\begin{tabular}{|c|c|c|c|c|c|c|}
\hline \multirow[b]{2}{*}{ Age } & \multicolumn{2}{|c|}{$\mathrm{T} 1^{2}$} & \multicolumn{2}{|c|}{$\mathrm{T} 2^{3}$} & \multicolumn{2}{|c|}{$\mathrm{T}^{4}$} \\
\hline & 24.0 & $(2.7)$ & 24.3 & $(2.9)$ & 25 & $(2.8)$ \\
\hline \multicolumn{7}{|l|}{ Sex } \\
\hline Female & 128 & $(92.7)$ & - & & - & \\
\hline Male & 10 & (7.3) & - & & - & \\
\hline \multicolumn{7}{|l|}{ Nursing education } \\
\hline University & 72 & $(52.2)$ & - & & - & \\
\hline Vocational school & 66 & $(47.8)$ & - & & - & \\
\hline \multicolumn{7}{|l|}{ Continued employment } \\
\hline Continuing to work in the same department/facility & - & & 138 & $(100.0)$ & 138 & $(100.0)$ \\
\hline Resignation, absence & - & & 0 & & 0 & \\
\hline \multicolumn{7}{|l|}{ Type of hospitals } \\
\hline Advanced care hospital & - & & 98 & $(71.1)$ & 98 & (71.1) \\
\hline Regional medical care facility & - & & 22 & $(15.9)$ & 22 & $(15.9)$ \\
\hline General hospital & & & 18 & $(13.0)$ & 18 & $(13.0)$ \\
\hline Clinic & - & & 0 & & 0 & \\
\hline \multicolumn{7}{|l|}{ Department } \\
\hline Ward & - & & 138 & $(100.0)$ & 138 & $(100.0)$ \\
\hline Out-patient clinic & - & & 0 & & 0 & \\
\hline
\end{tabular}

${ }^{1}$ Dara are the number (\%) or the mean value \pm standard deviation; ${ }^{2}$ Prior to employment; ${ }^{3} 3$ months after the employment; ${ }^{4} 1$ year after the employment.

higher $(\mathrm{p}=0.002)$ than that at T1. In other words, mental health improved to an extent from 3 months to 1 year after employment as a nurse, but 1 year after employment as a nurse it was worse than it was before employment.

\subsection{Variations in SOC upon Employment as a Nurse (Table 2)}

Each component of the SOC was examined. Sense of comprehensibility score decreased significantly upon employment as a nurse. Comparison of the score at different times indicated that the mean at T2 was signifycantly lower $(p<0.001)$ than that at T1. In addition, there were no significant variations in the score $(p=$ 0.143 ) from $\mathrm{T} 2$ to $\mathrm{T} 3$, but the mean at $\mathrm{T} 3$ was significantly lower $(p<0.001)$ than that at $\mathrm{T} 1$. After employment as a nurse, sense of comprehensibility remained lower than it was before employment.

The second component of the SOC is sense of manageability. Multiple comparison indicated that there were no significant differences in the score at $\mathrm{T} 1$ and $\mathrm{T} 2(\mathrm{p}=$ $0.517)$ and at $\mathrm{T} 2$ and $\mathrm{T} 3(\mathrm{p}=0.618)$, but the mean at T3 was significantly lower $(p=0.024)$ than that at T1. Sense of manageability gradually decreased in the year after employment as a nurse.

The third component of the SOC is sense of meaningfulness. Multiple comparison indicated that there were no significant differences in the score at T1 and T2 $(\mathrm{p}=$ $0.985)$, but the mean at T3 was significantly lower than that at T1 $(p<0.001)$ and T2 $(p=0.014)$. Sense of meaningfulness gradually decreased in the year after employment as a nurse.

\subsection{Effects of SOC on Variations in Mental Health (Tables 3(a), (b))}

The effects of SOC on variability in the GHQ score were studied in the 3 months after employment as a nurse. Sense of meaningfulness at T1 $(\beta=-0.386, \mathrm{p}<0.001)$, variability in sense of comprehensibility from $\mathrm{T} 1$ to $\mathrm{T} 2$ $(\beta=-0.421, \mathrm{p}<0.001)$, and variability in sense of manageability from T1 to T2 $(\beta=-0.182, \mathrm{p}=0.031)$ were significantly associated with variability in the GHQ score.

Similarly, the effects of SOC on variability in the GHQ score were studied from T2 to T3. Sense of meaningfulness at T3 $(\beta=-0.362, \mathrm{p}<0.001)$, variability in 
Table 2. Variations in mental health and SOC upon employment as a nurse ${ }^{1,2} \mathrm{n}=138$.

\begin{tabular}{|c|c|c|c|c|}
\hline & Mean & (SD) & & \\
\hline \multicolumn{5}{|c|}{ Mental health $(\mathrm{GHQ})^{4}$} \\
\hline $\mathrm{T} 1$ & 16.4 & $(6.2)$ & \multirow[t]{2}{*}{$<0.001$} & \multirow{3}{*}{0.002} \\
\hline $\mathrm{T} 2$ & 23.0 & $(7.2)$ & & \\
\hline $\mathrm{T} 3$ & 18.5 & $(6.3)$ & $<0.001$ & \\
\hline \multicolumn{5}{|c|}{ Sense of comprehensibility in SOC } \\
\hline $\mathrm{T} 1$ & 16.8 & $(3.8)$ & \multirow{2}{*}{$<0.001$} & \multirow{3}{*}{$<0.001$} \\
\hline $\mathrm{T} 2$ & 14.5 & $(4.4)$ & & \\
\hline $\mathrm{T} 3$ & 15.3 & (3.3) & 0.143 & \\
\hline \multicolumn{5}{|c|}{ Sense of manageability in SOC } \\
\hline $\mathrm{T} 1$ & 15.2 & $(3.7)$ & \multirow[t]{2}{*}{0.571} & \multirow{3}{*}{0.024} \\
\hline $\mathrm{T} 2$ & 14.7 & $(4.5)$ & & \\
\hline $\mathrm{T} 3$ & 14.3 & $(4.0)$ & 0.618 & \\
\hline \multicolumn{5}{|c|}{ Sense of meaningfulness in SOC } \\
\hline $\mathrm{T} 1$ & 17.5 & $(4.2)$ & \multirow[t]{2}{*}{0.985} & \multirow{3}{*}{0.001} \\
\hline $\mathrm{T} 2$ & 17.4 & $(4.3)$ & & \\
\hline $\mathrm{T} 3$ & 16.1 & $(4.7)$ & 0.014 & \\
\hline
\end{tabular}

${ }^{1}$ Analysis of variance (repeated measures) by a general linear model (covariates: age, sex, nursing education); ${ }^{2} \mathrm{~T} 1$ : Prior to employment, T2: 3 months after the employment, T3: 1 year after the employment; ${ }^{3}$ Multiple comparison by Bonferroni's technique; ${ }^{4} \mathrm{~A}$ higher score indicates poorer mental health.

sense of comprehensibility from T2 to T3 $(\beta=-0.335, \mathrm{p}$ $=0.014)$, variability in sense of manageability $(\beta=$ $-0.281, \mathrm{p}=0.035)$, and variability in sense of meaningfulness $(\beta=-0.359, \mathrm{p}<0.001)$ from T2 to T3 were significantly associated with variations in mental health.

\section{DISCUSSION}

\subsection{Variations in Mental Health upon Employment as a Nurse}

The current study found that new graduate nurses' mental health markedly worsens in the 3 months after their employment as nurses. This finding has been mentioned but not appropriately validated since previous studies used a baseline when employment had already begun. The current study used an online survey, which allowed respondents to be followed prior to their employment. This provided definite evidence of the actual worsening of new graduate nurses' mental health as had previously been mentioned.

Similarly, the current study found that new graduate nurses' mental health 1 year after their employment was worse than that before employment. Circumstances in
Japan are such that workers overall, including recent graduates, suffer poor mental health because of what is termed the "May blues" 2 - 3 months after employment, although this temporary condition is not regarded as depression with a transient adjustment disorder [28]. However, studies overseas [29] have focused on recent graduates' poor mental health during this period as a predictor of subsequent career development. The current study similarly indicated that worsening of mental health upon employment must not be disregarded as a temporary condition and must instead be observed over the long term.

\subsection{Variations in SOC upon Employment as a Nurse}

The changes in SOC in terms of each component revealed that all 3 components actually decreased in the year after employment as a nurse. Upon employment, new graduate nurses are placed in a completely different environment and must take on new tasks. Such major changes temporarily threaten their sense that the world they live in is coherent, and all of the perceptions that constitute their SOC are severely disturbed. Thus, new graduate nurses have an SOC that decreases or falters upon their employment. These nurses have diminished internal ability to cope with stressors and are susceptible to a stress response, which includes worsening of their mental health.

In adulthood, SOC is thought to be readily affected by work experience and subsequently develop into a fixed part of a person's core being [13]. The trends noted in the current study substantiate this theory. Many new graduate nurses are in early adulthood, which is when an SOC is being developed. At this age, the level of an individual's SOC is still in flux.

\subsection{Effects of SOC on Variations in Mental Health}

Results of the current study suggested internal approaches to foster the qualities of new graduate nurses with regard to coping with stressors in order to sustain and improve their mental health. This contention is supported by the findings of numerous studies of nurses who are not recent graduates [16-22] and workers other than nurses [30-32]. These findings corroborate the fact that SOC is an important concept for new graduate nurses to actively work while exposed to a great deal of occupational stressor.

The current study also found that the SOC that somehow develops after employment is more important than the SOC at one time in terms of sustaining and improving mental health. Work experience is what most affects the development of SOC during adulthood. During this 
Table 3. (a) Effects of SOC on variations in mental health (from T1 to T2) ${ }^{1,2,3,4} \mathrm{n}=138$; (b) Effects of SOC on variations in mental health (from T2 to T3) ) $^{1,2,3,4} \mathrm{n}=138$.

(a)

\begin{tabular}{ccccc}
\hline & $\mathrm{B}$ & $\mathrm{SE}$ & $\beta$ & $\mathrm{p}$ \\
\hline T1 Sense of comprehensibility & -0.250 & 0.297 & -0.102 & 0.400 \\
T1 Sense of manageability & -0.107 & 0.295 & -0.043 & 0.518 \\
T1 Sense of meaningfulness & -0.625 & 0.214 & -0.386 & $<0.001$ \\
From T1 to T2 Sense of comprehensibility & -0.753 & 0.206 & -0.421 & $<0.001$ \\
From T1 to T2 Sense of manageability & -0.397 & 0.182 & -0.182 & 0.031 \\
From T1 to T2 Sense of meaningfulness & -0.281 & 0.192 & -0.122 & 0.147 \\
Adjusted $\mathrm{R}^{2}$ value & 0.464 & & &
\end{tabular}

${ }^{1}$ Multiple regression analysis; ${ }^{2}$ Dependent variable: GHQ score from T1 to T2 (a higher score indicates worsening of mental health); ${ }^{3}$ Contorol variable: age, sex, nursing education; ${ }^{4} \mathrm{~T} 1$ : Prior to employment, T2: 3 months after the employment.

(b)

\begin{tabular}{ccccc}
\hline & $\mathrm{B}$ & $\mathrm{SE}$ & $\beta$ & $\mathrm{p}$ \\
\hline T2 Sense of comprehensibility & -0.237 & 0.175 & -0.114 & 0.268 \\
T2 Sense of manageability & -0.200 & 0.186 & -0.089 & 0.303 \\
T2 Sense of meaningfulness & -0.631 & 0.225 & -0.362 & $<0.001$ \\
From T2 to T3 Sense of comprehensibility & -0.590 & 0.238 & -0.335 & 0.014 \\
From T2 to T3 Sense of manageability & -0.466 & 0.242 & -0.281 & 0.035 \\
From T2 to T3 Sense of meaningfulness & -0.561 & 0.148 & -0.359 & $<0.001$ \\
Adjusted $\mathrm{R}^{2}$ value & 0.439 & & & \\
\hline
\end{tabular}

${ }^{1}$ Multiple regression analysis; ${ }^{2}$ Dependent variable: GHQ score from T2 to T3 (a higher score indicates worsening of mental health); ${ }^{3}$ Contorol variable: age, sex, nursing education; ${ }^{4} \mathrm{~T} 2: 3$ months after the employment, T3: 1 year after the employment.

period, SOC is greatly affected by workplace conditions such as experience with success at work $[13,14]$. The SOC of new graduate nurses decreases after their employment and subsequently remains low, so continuing efforts must be made in the workplace to provide them with quality work experience and encourage them to develop SOC.

Many previous studies have directly linked worsening of new graduate nurses' mental health (poor mental health at one time, strictly speaking) to occupational stressor. However, results of the current study suggested that variations in mental health upon employment, which many new graduate nurses experience, are not simply a result of occupational stressor. Rather, those variations are a complex phenomenon produced by their SOC decreasing or faltering upon their employment and their inability to combat occupational stressor. In nursing, occupational stressor cannot be avoided to some extent. External approaches to reduce occupational stressor as much as possible and internal approaches to foster the qualities of new graduate nurses with regard to coping with stressor are both crucial.

\subsection{Practical Implications}

First, current study suggested the importance of efforts to sustain and improve the mental health of new graduate nurses. Current support measures for new graduate nurses at medical facilities in Japan tend to be biased toward skill and knowledge education and training. According to a report on a survey of nursing administrators at medical facilities regarding the implementation of support measures for new graduate nurses [33], 80.8\% of medical facilities had an "educational/training program for practical skills". Only $38.6 \%$ of medical facilities "provided a system of mental health measures" and the remaining $39.5 \%$ had "no plans to do so in the future". Medical facilities in Japan must be fully cognizant of the problem of worsening of new graduate nurses' mental health, and sustaining and improving mental health must be regarded as a key goal of support for new graduate nurses.

Second, the current study suggested that development of new graduate nurses' SOC must be encouraged. Although there are few empirical studies, several previous 
studies have indicated a potential path to creating a workplace that effectively encourages development of an SOC. Meaningfulness is the core component of SOC with the greatest ability to affect variations in mental health. External approaches that give meaning to one's work are crucial to facilitating a sense of meaningfulness $[13,14]$. Meaning can be entirely subjective; more important is the sense of "finding meaning". Conventional approaches for new graduate nurses use various means to teach work norms. They tend to impart negative or positive values regarding nursing, e.g. "nursing is a very rewarding job in which one is responsible for people's lives". An approach that only imposes "normative values" regarding work cannot be expected to facilitate a sense of meaningfulness. Drawing on the personal values of each new graduate nurse is crucial to facilitating a sense of meaningfulness. New graduate nurses must be given the latitude to find some meaning in their work even if that meaning appears negative, e.g. "nursing is a necessary job that allows one to make a living". Both pre- and post-employment interventions must be used to facilitate that personal search for meaning. Basic nursing education before employment must also encourage students to find personal meaning in nursing as a profession, and opportunities for career counseling and feedback must be provided after employment to give new nurses a feel for that profession.

\subsection{Limitations of This Study}

The design of the current study allowed participation by individuals who quit or took a leave of absence, but actual study participants were limited to individuals who continued to work 1 year after their employment as nurses. In addition, respondents in the current study were all assigned to wards of hospitals in the Kanto region. The characteristics of these respondents may have led to bias in mental health (SOC) and the level of desire to stay in one's profession, and the study had a relatively small sample size, so caution is required with regard to generalizing the results obtained. In the future, the sample must be expanded to varied regions and facilities and include individuals who quit. Findings that account for the reality faced by new graduate nurses in Japan must also be obtained.

\section{CONCLUSIONS}

The current research conducted a longitudinal study of new graduate nurses at 3 times - prior to employment as a nurse and 3 months and 1 year afterwards. This study yielded 3 findings as noted below.

First, new graduate nurses' mental health significantly worsened 3 months after their employment as nurses, and mental health 1 year after employment was significantly worse than that before employment. Second, all the 3 components of SOC decreased significantly in the year after employment as a nurse. And third, the mental health of new graduate nurses was significantly affected by low SOC and by previous diminishing of the SOC.

\section{ACKNOWLEDGMENTS}

This study was conducted as part of basic research (A) under a Grantin-Aid for Scientific Research from the Ministry of Education, Culture, Sports, Science and Technology (Project No. 21243033). The authors wish to thank all of the respondents to the 3 surveys and study supervisors at the educational institutions surveyed.

\section{REFERENCES}

[1] Ministry of Health, Labour and Welfare (2010) Review panel report on the 7th nursing personnel supply and demand projections. (in Japanese)

http://www.mhlw.go.jp/stf/houdou/2r9852000000z68f-im g/2r9852000000z6df.pdf

[2] Mizuta, M., Kousaka, R., Tsuji, S., Nakano M. and Inoue J. (2004) New graduate nurses' mental health and desire to leave the nursing profession. Nursing College Bulletin, Wakayama Medical University, 7, 21-27. (in Japanese)

[3] Kobayashi, C. and Nakamura, M. (2007) Occupational stress and coping behaviors of new graduate nurses. $\mathrm{Ya}$ manashi Nursing Journal, 7, 13-20. (in Japanese)

[4] Nakamura, R., Murata, C. and Takahashi, S. (2006) A study of support for new graduate nurses to adjust to the workplace. Nursing Bulletin of Hirosaki University, 1, 41-50. (in Japanese)

[5] Ohtsubo, T. and Kohda, R. (2003) Stress management for nursing: A psychiatrist's view of stress management for nursing in Japan. International Nursing Review, 26, $47-$ 51. (in Japanese)

[6] Morimoto, H., Mizuko, M., Minakami, K., Katayama, H. and Iwabuchi, C. (2005) Research on a nurse's mental health. Journal of Kawasaki Medical Welfare Society, 15, 243-247.

[7] Onizawa, N. and Matsunaga, Y. (2011) Relationship between accumulate fatigue and willingness of occupation continuation. Journal of Japanese Nursing Research, 34, 45-53.

[8] Tei-Tominaga, M., Miki, A. and Fujimura, K. (2009) A cross-sectional study of factors associated with intensions to leave among newly graduated nurses in eight advanced treatment hospitals: Job stress, job readiness, and subjective health status. Japan Journal of Public Health, 56, 301-311.

[9] McNeely, E. (2005) The consequences of job stress for nurses' health. Nursing Outlook, 53, 291-299. doi:10.1016/j.outlook.2005.10.001

[10] McVicar, A. (2003) Workplace stress in nursing: A literature review. Journal of Advanced Nursing, 44, 633642. doi:10.1046/i.0309-2402.2003.02853.x

[11] Motoaki, H., Oeda, M. and Haruki, Y. (1991) Psychology 
of stress: Cognitive appraisal and coping research. Jitsumukyoiku-Shuppan, Tokyo.

[12] Sarid, O., Berger, R. and Segal-Engelchin, D. (2010) The impact of cognitive behavioral interventions on SOC, perceived stress and mood states of nurse. Procedia Social and Behavioral Sciences, 2, 928-932.

[13] Antonovsky, A. (1987) Unraveling the mystery of health: How people manage stress and stray well. Jossey-Bass Publishers, San Francisco.

[14] Yamazaki, Y., Togari, T. and Sakano, J., Eds. (2008) The ability to cope with stress: SOC. Yushindo, Tokyo. (in Japanese)

[15] Togari T. (2009) Developments in and topics of SOC research in nursing. Journal of the Japan Society of Nursing Research, 42, 491-503. (in Japanese)

[16] Iwatani, M., Watanabe, K. and Kunikata, H. (2008) A study on the mental health of critical care nurses: In relation to emotional labor, a sense of coherence, and stress response. Journal of the Japan Society of Nursing Research, 31, 87-93. (in Japanese)

[17] Eda, S., Tatsumi, Y. and Nomura, M. (2007) Association between emergency nurses' sense of coherence and burnout due to stress. Journal of the Japanese Association for Emergency Nursing, 8, 32-42. (in Japanese)

[18] Watanabe, T., Shigehisa, K., Koiso, R. and Tokuse, Y. (2007) Association between nurses' stress and work specialization. Japanese Journal of Nursing Management, 17, 871-876. (in Japanese)

[19] Nakanishi, M., Tsuge, Y. and Futatsumori, E. (2008) Relationship between the desire to stay in nursing of midlevel nurses at 5 affiliated hospitals and their job satisfaction and sense of coherence (SOC). Proceedings of the Japanese Nursing Association: Nursing Management, 38, 139-141. (in Japanese)

[20] Shiu, A.T. (1998) The significance of sense of coherence for the perceptions of task characteristics and stress during interrupting amongst a sample of public health nurses in Hong Kong. Public Health Nursing, 15, 273-280. doi:10.1111/j.1525-1446.1998.tb00350.x

[21] Takeuchi, T. and Yamazaki, Y. (2010) Relationship between work-family conflict and sense of coherence among Japanese registered nurses. Japan Journal of Nursing Science, 7, 158-168. doi:10.1111/j.1742-7924.2010.00154.x
[22] Takeuchi, T., Togari, T. and Yamazaki, Y. (2009) Nurses' SOC and nature of the workplace. Journal of the Japan Society of Nursing Research, 42, 517-526. (in Japanese)

[23] Goldberg, D.P. and Blackwell, B. (1970) Psychiatric illness in general practice: A detailed study using a new method of case identification. British Medical Journal, 1, 439-443. doi:10.1136/bmj.2.5707.439

[24] Nakagawa, Y. and Daibo, I. (1985) Manual for the Japanese version of the GHQ. Nihon Bunka Kagakusha, Tokyo. (in Japanese)

[25] Niiro, M. and Mori, T. (2001) Examination of the reliability and validity of the Japanese version of the 12-item GHQ 12 (GHQ-12) based on a survey of corporate employees. Clinical Psychiatry, 43, 431-436. (in Japanese)

[26] Antonovsky, A. (1993) The structure and properties of the sense of coherence scale. Social Science and Medicine, 36, 275-733. doi:10.1016/0277-9536(93)90033-Z

[27] Yamazaki, Y. (1999) A new view of health provided by salutogenesis and a sense of coherence (SOC). Quality Nursing, 5, 825-832. (in Japanese)

[28] Shima, S., Shikano, T., Kitamura, T. and Asai, M. (1985) A new self-rating depression scale. Clinical Psychiatry, 27, 717-723. (in Japanese)

[29] Cooper-Thomas, H. and Anderson, N. (2002) Newcomer adjustment: The relationship between organizational socialization tactics, information acquisition and attitudes. Journal of Occupational and Organizational Psychology, 75, 423-437. doi:10.1348/096317902321119583

[30] Gilbar, O. (1998) Relationship between burnout and sense of coherence in health workers. Social Work in Health Care, 26, 39-49. doi:10.1300/J010v26n03 03

[31] Baker, M., North, D. and Smith, D.F. (1997) Burnout, sense of coherence and sources of salutogenesis in social workers. Journal of Human Behavior, 34, 22-66.

[32] Palsson, M.B., Hallberg, J.R., Norberg, A. and Björvell, H. (1997) Burnout, empathy, and sense of coherence among Swedish district nurses before and after systematic clinical supervision. Scandinavian Journal of Caring Science, 10, 21-26.

[33] Central Nursing Center, Japanese Nursing Association (2005) Report on measures to prevent early departure of new nursing personnel graduating in 2005. (in Japanese) https://www.nurse-center.net/html1/sm01/SM010801 S1 701.html 